and not only cancers but also diseases related to reproductive toxicity such as spontaneous abortion and others. Further, we're planning to establish a monitoring and surveillance system for the known and the unknown hazards like unexpected by-products. We were approved from Korea National Institute for Bioethics Policy (KoNIBP) for cohort establishment in July 2017. We held cohort information session of over two hundred times for entire employees. In these sessions, the employees were encouraged to participate to the cohort study with full explanation related to the establishment of cohort system including the necessity, value, and vision of the system. The consent form was included specific sub-categories depending on the characteristics and source of personal and sensitive information to be used. A total of 22490 employees were agreed to participate to SHE (SKHynix Employees) cohort (the participation rate: 93 percent) and we built up the foundation of the cohort with these higher rates. Various data included in the consent form, such as personnel information, health examination information, and job exposure matrix (JEM) and others were collected and refined to identify health status of our employees. The cohort was built on the basis of the need of our own healthcare system, and the establishment and the analyses of cohort DB are expected to deduct of the high risk group through tracking and monitoring for risk factors and major diseases. It is also expected proactive prevention through blocking potential risks.

\section{P.1.23 GAPS BETWEEN INTERNATIONAL CONVENTIONS AND NATIONAL ASBESTOS BAN POLICIES: A GLOBAL PERSPECTIVE}

Ro-ting Lin. China Medical University, Taichung, Taiwan

\subsection{6/OEM-2019-EPI.225}

Introduction There is an international consensus that the most efficient way to eliminate asbestos-related diseases is to stop using asbestos. However, $80 \%$ of the global population lives in countries that lack any national policy that achieves a total ban on asbestos. We investigated whether national policies were in line with international conventions and identified countries that lag behind the global trend toward a total asbestos ban.

Methods We obtained data on the year that each country implemented policies that ban chrysotile, crocidolite, and amosite. We also obtained the list of countries that have ratified the C162 Asbestos Convention and the Basel Convention, and their date of ratification. Data retrieved from the search databases were compiled for statistical analysis. We calculated the numbers and proportions of countries enforcing total bans, partial bans, and no bans.

Results As of 2018, there were 62 countries implemented the total asbestos ban policy. Countries that ratified both conventions and countries that ratified either one or unratified any convention, we found the former group had a higher proportion of implementing total ban compared to the latter group $(65 \%$ vs. $41 \%$, p-value $<0.05)$. However, some countries, such as Russia and Kazakhstan, are still among the biggest producers of asbestos, despite they have ratified C162. Asbestos may have been consumed longer in countries not ratifying conventions than those ratifying conventions.

Conclusions Historical asbestos consumption and loose regulations have introduced challenges to eliminate asbestos-related diseases. Although several countries have reoriented the national policy in line with the global trend of banning all types and forms of asbestos, the gap between adopting interventional conventions and developing national policies remain, warranting efforts to analyze driving forces behind successful political processes to ratify the international conventions, establish asbestos control limits, and eventually create a national policy for a total asbestos ban.

\section{P.1.24 THE DEVELOPMENT AND IMPACT OF POLICIES AND RESEARCH FOR PREVENTING AND RECOGNIZING OVERWORK-RELATED CARDIOVASCULAR DISEASE IN TAIWAN}

Ro-ting Lin. China Medical University, Taichung, Taiwan

\subsection{6/OEM-2019-EPI.226}

Introduction Overwork-related cardiovascular and cerebrovascular disease (CVD) is an emerging occupational and public health issue in East Asian societies with a substantial impact on workers' rights and labor standards. To eliminate exposure to work-related risk factors and prevent overworkrelated CVD, establishing national policy is a key step. We investigated the development and impact of policies and research regarding overwork-related CVD in recent years in Taiwan.

Methods We collected information regarding government policies that aimed to prevent and recognize overworkrelated CVD. Among the risk factors, long working hours have been widely adopted as a quantitative measure to recognize CVD as overwork-related or not. We also collected data on the number of overwork-related CVD cases and average working hours for each industry from 2006 to 2017.

Results In Taiwan, overwork-related CVD 79\% of all deaths due to occupational diseases in 2017, although it accounted for $13 \%$ of all cases of occupational diseases. Taiwanese workers have long working hours compared to those in other countries, but Taiwan did not have official criteria for recognizing overwork-related diseases until 1991, and it did not recognize its first case until 2006 after several onsets of CVD reported on the news. Our estimation showed an under-recognition problem in less severe outcomes (i.e., illness). We also found variations in CVD risks across industries, with the higher risk in transportation and information.

Conclusions National policy changes for preventing overwork were pushed by insightful scholars, labor unions, nongovernmental organizations, and legislators. Clear criteria for recognizing overwork-related CVD can help occupational physicians and industrial hygienists to assess the workers' working characteristics more objectively. Although Taiwanese government has adopted the criteria for recognizing overwork-related CVD from Japan, the underreporting of less severe outcomes and industry disparities warrants further research to explore causal mechanisms and policies to narrow the gaps. 